\title{
ORIENTATIONAL EFFECTS IN ELECTRIC-FIELD-INDUCED SPECTRA
}

\author{
David L. ANDREWS and B.S. SHERBORNE \\ School of Chemical Sciences, University of East Anglia, Norwich NR47TJ, UK
}

Received 2 June 1986

\begin{abstract}
The electrochromic effect of a static electric field on the rate of absorption of electromagnetic radiation by a molecular fluid is investigated within the framework of quantum electrodynamics. The anisotropic, Boltzmann-weighted orientation of polar molecules in a fluid is treated using an exact method, giving a set of expressions that include both the optical and electro-optical pathways. Using irreducible tensor methods the complete set of rate equations is presented, and symmetry implications are analysed in detail. Polarisation dependence is also explicitly included in the results, and the likely orders of magnitude of the various rate contributions are estimated.
\end{abstract}

\section{Introduction}

Application of a static electric field to molecular systems can induce changes in their absorption spectra via a number of different mechanisms. Such phenomena, which fall under the general heading of electrochromism, are frequently studied in the solid state where the molecules have a comparatively fixed orientation. The appropriate selection rules and polarisation dependence have recently been summarised by Stedman [1]. In the case of fluid media, the theory is less well established, and in a recent paper [2] we have developed a theory to describe optical transitions induced in free molecules by a static electric field. This theory was simplified by assuming an isotropic distribution of randomly oriented non-polar molecules, and attention was focused on transitions which are electric dipole (E1) forbidden in the absence of an electric field. In this paper we take the general case in which the molecules may possess a permanent electric dipole moment, which leads to a degree of alignment with the applied field, and we relax the earlier restriction to (E1)forbidden transitions.

In order to investigate the orientational effects resulting from application of the electric field, we have made use of recently developed techniques $[3,4]$ which allow an exact treatment of the aniso- tropic Boltzmann-weighted averages required. Our results are obtained within the framework of molecular quantum electrodynamics (QED) [5], and in contrast to earlier treatments [6] incorporate those terms which arise through quantum interference between the optical (E1) and field-induced electro-optical (E1 $\times$ E1) excitation channels. Temperature, field and polarisation dependence are explicitly given in these results, and the selection rules are examined in detail. Finally, an estimate is given of the likely orders of magnitude of the effects discussed.

\section{Quantum formalism}

We can begin by deriving the matrix element for single-photon absorption using the quantum electrodynamical perturbation operator

$\mathscr{H}_{\text {int }}=-\epsilon_{0}^{-1} \boldsymbol{\mu} \cdot \boldsymbol{d}^{\perp}-\mu \cdot \mathscr{E}$,

where $\mu$ is the electric dipole moment operator, $d^{\perp}$ is the transverse electric displacement operator for the radiation field, and $\mathscr{E}$ is the static electric field. This operates on the eigenstates of the unperturbed molecular and radiation hamiltonians, although it can be shown [2] that an alternative approach, using molecular states dressed by the static field, and the first term of (1) for the 
coupling leads to the same solutions. Using the implied summation convention for repeated tensor indices, the leading terms in the matrix element which result from first- and second-order perturbation theory, can be written as follows

$M_{f 0}=-\mathrm{i}\left(n h \omega / 2 \epsilon_{0} V\right)^{1 / 2}\left(\mu_{i}^{f 0} e_{j}-S_{i j}^{f 0} \mathscr{E}_{i} e_{j}+\cdots\right)$,

where $n$ is the number of photons within the quantisation volume $V, \omega$ is the circular frequency of the radiation satisfying the condition

$\hbar \omega=E_{f}-E_{0}$,

$\mu^{f 0}$ is the transition electric dipole moment, $e$ is the polarisation vector for the radiation, which may be complex to admit the possibility of circular polarisations, and $S_{i j}^{f 0}$ is a molecular response tensor defined by

$S_{i j}^{f 0}=\sum_{r}\left[\mu_{i}^{f r} \mu_{j}^{r 0} /\left(E_{0 r}+\hbar c k\right)+\mu_{j}^{f r} \mu_{i}^{r 0} / E_{0 r}\right]$.

The first term in eq. (2) can be identified with the time-ordered diagram (a) shown in fig. 1; the second term can be represented by the timeordered diagrams (b) and (c), corresponding to the two parts of the tensor $S_{i j}^{f 0}$ defined by eq. (4). It should be noted that this tensor may exhibit resonant enhancement in cases where a near degenerate state, $|v\rangle$, exists close in energy to the final or initial states, provided that the transitions $|f\rangle \leftarrow$ $|v\rangle$, and $|v\rangle \leftarrow|0\rangle$ are (E1)-allowed.

The rate of absorption can now be obtained from eq. (2) using the Fermi golden rule; the principal terms are as follows

$$
\begin{aligned}
\Gamma= & \left(n \pi \omega \rho_{\mathrm{f}} / \epsilon_{0} V\right)\left(\mu_{i}^{f 0} \mu_{j}^{f 0} e_{i} \bar{e}_{j}-\mu_{i}^{f 0} S_{j k}^{f 0} e_{i} \mathscr{E}_{j} \bar{e}_{k}\right. \\
& \left.-\mu_{i}^{f 0} S_{j k}^{f 0} \bar{e}_{i} \mathscr{E}_{j} e_{k}+S_{i j}^{f 0} S_{k l}^{f 0} \mathscr{E}_{i} e_{j} \mathscr{E}_{k} \bar{e}_{l}+\cdots\right),
\end{aligned}
$$

in which $\rho_{\mathrm{f}}$ is the density of molecular final states. Finally, the result can be recast in terms of the incident irradiance $I$, through the relation

$I=n \hbar c \omega / V$,

leading to

$$
\begin{aligned}
\Gamma= & \left(\pi I \rho_{\mathrm{f}} / \epsilon_{0} \hbar c\right)\left\langle\mu_{i}^{f 0} \mu_{j}^{f 0} e_{i} \bar{e}_{j}-\mu_{i}^{f 0} S_{j k}^{f 0} \mathscr{E}_{j}\right. \\
& \left.\times\left(e_{i} \bar{e}_{k}+\bar{e}_{i} e_{k}\right)+S_{i j}^{f 0} S_{k l}^{f 0} \mathscr{E}_{i} e_{j} \mathscr{E}_{k} \bar{e}_{l}\right\rangle,
\end{aligned}
$$

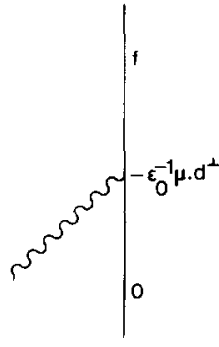

(a)

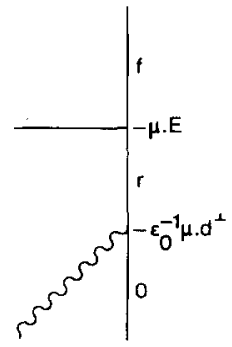

(b)

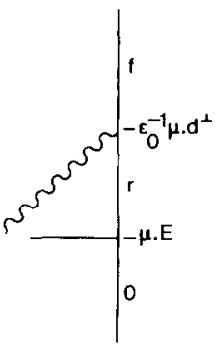

(c)
Fig. 1. The three time-ordered diagrams for electric-field induced absorption; (a) represents the optically (E1)-allowed channel and (b), (c) together represent the electro-optically $($ E1 $\times$ E1)-allowed channel.

in which the angular brackets denote the rotational averaging required to account for the distribution of molecular orientations in any fluid sample.

\section{Boltzmann-weighted orientational averages}

The rotational average in eq. (7) has to be effected with regard to an anisotropic orientational distribution. This results from the preferential alignment of the polar molecules with the applied static field, determined by the torque $\mu^{00}$ $\times \mathscr{E}$, where $\mu^{00}$ is the static electric dipole moment. Any molecular orientation by interaction with the electric field of the radiation will be negligible.

By weighting the averages in eq. (7) with the Boltzmann term $\exp \left(\mu^{00} \cdot \mathscr{E} / k T\right)$ to allow for the anisotropic distribution, the following expression for the rate is obtained

$$
\begin{aligned}
\Gamma= & \left(\pi I \rho_{l} / \varepsilon h c\right)\left\langle\left[\mu_{i}^{f 0} \mu_{j}^{f 0} e_{i} \bar{e}_{j}-\mu_{i}^{f 0} S_{j k}^{f 0} \mathscr{E}_{j}\left(e_{i} \bar{e}_{k}+\bar{e}_{i} e_{k}\right)\right.\right. \\
& \left.\left.+S_{i j}^{f 0} S_{k l}^{f 0} \mathscr{E}_{i} e_{j} \mathscr{E}_{k} \bar{e}_{l}\right] \exp \left(\mu^{00} \cdot \mathscr{E} / k T\right)\right\rangle \\
& \times\left\langle\exp \left(\mu^{00} \cdot \mathscr{E} / k T\right)\right\rangle^{-1}
\end{aligned}
$$

The weighted average in eq. (8) can be approximated by expanding the exponential and retaining only the linear terms. However, it has recently been shown that an exact result in terms of spheri- 
cal Bessel functions can be obtained [3] by referring all molecular parameters to a molecule-fixed cartesian frame (denoted by Greek indices) and averaging over the direction cosines between axes in the laboratory-fixed and molecule-fixed frames. Thus if $l_{i \lambda}$ denotes the direction cosine between the $i$-axis in the laboratory frame and the $\lambda$-axis in the molecular frame, the expression becomes

$$
\begin{aligned}
\Gamma= & \left(\pi I \rho_{\mathrm{f}} / \epsilon_{0} \hbar c\right)\left[\mu_{\lambda}^{f 0} \mu_{\mu}^{f 0} e_{i} \bar{e}_{j}\right. \\
& \times\left\langle l_{i \lambda} l_{j \mu} \exp \left(\mu_{\rho}^{00} \mathscr{E}_{n} l_{n \rho} / k T\right)\right\rangle \\
& -\mu_{\lambda}^{f 0} S_{\mu \nu}^{f 0} \mathscr{E}_{j}\left(e_{i} \bar{e}_{k}+\bar{e}_{i} e_{k}\right) \\
& \times\left\langle l_{i \lambda} l_{j \mu} l_{k \nu} \exp \left(\mu_{\rho}^{00} \mathscr{E}_{n} l_{n \rho} / k T\right)\right\rangle \\
& +S_{\lambda \mu}^{00} S_{\nu \mathrm{o}}^{f 0} \mathscr{E}_{i} e_{j} \mathscr{E}_{k} \bar{e}_{l} \\
& \left.\times\left\langle l_{i \lambda} l_{j \mu} l_{k \nu} l_{l o} \exp \left(\mu_{\rho}^{00} \mathscr{E}_{n} l_{n \rho} / k T\right)\right\rangle\right] \\
& \times\left\langle\exp \left(\mu_{\sigma}^{00} \mathscr{E}_{p} l_{p o} / k T\right)\right\rangle^{-1},
\end{aligned}
$$

which can be expressed

$$
\begin{aligned}
\Gamma= & \left(\pi I \rho_{\mathrm{f}} / \epsilon_{0} \hbar c\right)\left[\mu_{\lambda}^{f 0} \mu_{\mu}^{f 0} e_{i} \bar{e}_{j} I_{i j ; \lambda \mu}^{(2) \phi}\left(-\mathrm{i} \gamma, \hat{\mathscr{E}}, \hat{\mu}^{00}\right)\right. \\
& -\mu_{\lambda}^{f 0} S_{\mu \nu}^{f 0} \mathscr{E}_{j}\left(e_{i} \bar{e}_{k}+\bar{e}_{i} e_{k}\right) I_{i j k ; \lambda \mu \nu}^{(3) \phi}\left(-\mathrm{i} \gamma, \hat{\mathscr{E}}, \hat{\mu}^{00}\right) \\
& \left.+S_{\lambda \mu}^{f 0} S_{\nu 0}^{f 0} e_{i} \mathscr{E}_{j} \bar{e}_{k} \mathscr{E}_{l} I_{i j k i ; \lambda \mu \nu \text { o }}^{(4) \phi}\left(-\mathrm{i} \gamma, \hat{\mathscr{E}}, \hat{\mu}^{00}\right)\right] \\
& \times\left[I^{(0) \phi}\left(-\mathrm{i} \gamma, \hat{E}^{0}, \hat{\mu}^{00}\right)\right]^{-1},
\end{aligned}
$$

where

$\gamma=\mu^{00} \mathscr{E} / k T$,

and the results for the weighted averages are given in ref. [3].

\section{Results}

By application of the results for the weighted averages in eq. (10) we obtain an expression consisting of three sets of terms. The first set, $A$, results from the average of the leading $\mu_{\lambda}^{f 0} \mu_{\mu}^{f 0}$ term, and corresponds to optically (E1)-allowed transitions. The second set of terms, $B$, results from quantum interference between the optically and electro-optically allowed routes to the final state, and thus applies only to transitions which are both (E1)- and (E1 $\times$ E1)-allowed. Finally the third set, $C$, results from the average of the $S_{\lambda_{\mu}}^{f} S_{\nu \mathrm{o}}^{f 0}$ electro-optical term, and thus persists for any transition which is $(E 1 \times$ E1)-allowed. The detailed results are as follows:

$\Gamma=\left(\pi I \rho_{\mathrm{f}} / \epsilon_{0} \hbar c\right)(A+B+C)$.

where

$$
\begin{aligned}
A= & \frac{1}{3}\left(\mu_{\lambda}^{f 0} \mu_{\lambda}^{f 0}\right)+\frac{1}{6} j_{2}^{\prime}(-\mathrm{i} \gamma)(3 p-1)\left[\left(\mu_{\lambda}^{f 0} \mu_{\lambda}^{f 0}\right)\right. \\
& \left.-3\left(\mu_{\lambda}^{f 0} \hat{\mu}_{\lambda}^{00}\right)\left(\mu_{\mu}^{f 0} \hat{\mu}_{\mu}^{00}\right)\right] \\
B= & -\frac{1}{5} \mathrm{i} j_{1}^{\prime}(-\mathrm{i} \gamma) \mathscr{E}^{\circ}\left[(3 p-1)\left(S_{\lambda \nu}^{f 0} \mu_{\lambda}^{f 0}+S_{\lambda \lambda}^{f 0} \mu_{\nu}^{f 0}\right)\right. \\
& \left.+2(2-p)\left(S_{\nu \lambda}^{f 0} \mu_{\lambda}^{f 0}\right)\right] \hat{\mu}_{\nu}^{00} \\
& +\frac{1}{5} \mathrm{i} j_{3}^{\prime}(-\mathrm{i} \gamma) \mathscr{E}(3 p-1)\left[5 \mu_{\lambda}^{f 0} \hat{\mu}_{\lambda}^{00} S_{\mu \nu}^{f 0} \hat{\mu}_{\mu}^{00}\right. \\
& \left.-\left(S_{\lambda \nu}^{f 0} \mu_{\lambda}^{f 0}+S_{\nu \lambda}^{f 0} \mu_{\lambda}^{f 0}+S_{\lambda \lambda}^{f 0} \mu_{\nu}^{f 0}\right)\right] \hat{\mu}_{\nu}^{00}, \\
C= & \frac{1}{30} \mathscr{E}^{2}\left[(3 p-1)\left(S_{\lambda \lambda}^{f 0} S_{\mu \mu}^{f 0}+S_{\lambda \mu}^{f 0} S_{\mu \lambda}^{f 0}\right)\right. \\
& \left.+2(2-p)\left(S_{\lambda \mu}^{f 0} S_{\lambda \mu}^{f 0}\right)\right] \\
& -\frac{1}{42} j_{2}^{\prime}(-\mathrm{i} \gamma) \mathscr{E}^{2}\left[2 ( 3 p - 5 ) \left(3 S_{\lambda \mu}^{f 0} S_{\nu \nu}^{f 0} \hat{\mu}_{\lambda}^{00} \hat{\mu}_{\mu}^{00}\right.\right. \\
& \left.+3 S_{\nu \lambda}^{f 0} S_{\mu \nu}^{f 0} \hat{\mu}_{\lambda}^{00} \hat{\mu}_{\mu}^{00}-S_{\lambda \lambda}^{f 0} S_{\mu \mu}^{f 0}-S_{\lambda \mu}^{f 0} S_{\mu \lambda}^{f 0}\right) \\
& +3(3 p-1)\left(S_{\nu \lambda}^{f 0} S_{\nu \mu}^{f 0} \hat{\mu}_{\lambda}^{00} \hat{\mu}_{\mu}^{00}\right)+6(3-2 p) \\
& \left.\times\left(S_{\lambda \nu}^{f 0} S_{\mu \nu}^{f 0} \hat{\mu}_{\lambda}^{00} \hat{\mu}_{\mu}^{00}\right)+(p-5)\left(S_{\lambda \mu}^{f 0} S_{\lambda \mu}^{f 0}\right)\right] \\
& +\frac{1}{35} j_{4}^{\prime}(-\mathrm{i} \gamma) \mathscr{E}^{\circ 2}(3 p-1)\left\{\left[35 S_{\lambda \mu}^{f 0} S_{\nu o}^{f 0} \hat{\mu}_{\nu}^{00} \hat{\mu}_{o}^{00}\right.\right. \\
& -5\left(2 S_{\nu \nu}^{f 0} S_{\lambda \mu}^{00}+2 S_{\nu \lambda}^{f 0} S_{\mu \nu}^{f 0}+S_{\nu \lambda}^{f 0} S_{\nu \mu}^{f 0}\right. \\
& \left.\left.+S_{\lambda \nu}^{f 0} S_{\mu \nu}^{f 0}\right)\right] \hat{\mu}_{\lambda}^{00} \hat{\mu}_{\mu}^{00} \\
& \left.+\left(S_{\lambda \lambda}^{f 0} S_{\mu \mu}^{f 0}+S_{\lambda \mu}^{f 0} S_{\lambda \mu}^{f 0}+S_{\lambda \mu}^{f 0} S_{\mu \lambda}^{f 0}\right)\right\} \\
& +15)
\end{aligned}
$$

and where the polarisation parameter $p$ is defined by

$$
p=(\mathscr{E} \cdot e)(\hat{E} \cdot \bar{e}) \text {. }
$$

The reduced spherical Bessel functions $j_{n}^{\prime}(-i \gamma)$ are defined in terms of the spherical Bessel functions $j_{n}(-\mathrm{i} \gamma)$ as follows

$j_{n}^{\prime}(-\mathrm{i} \gamma)=j_{n}(-\mathrm{i} \gamma) / j_{0}(-\mathrm{i} \gamma)$,

the general expansions of which are given in table 1 , along with the high-temperature limit, $\gamma \ll 1$, and for completeness the low-temperature limit, $\gamma \gg 1$. It may be useful in assessing the relative 
importance of the terms $j_{n}^{\prime}(-i \gamma)$, to note that in the high-temperature limit, the leading terms can be written as

$j_{n}^{\prime}(-\mathrm{i} \gamma) \approx(-\mathrm{i} \gamma)^{n} /(2 n+1) ! !$.

Investigation of the detailed symmetry properties of the rate can be made easier by introducing irreducible tensors [7]. A second-rank cartesian tensor can be decomposed as follows

$S_{\lambda \mu}^{f 0}=S_{\lambda \mu}^{(0)}+S_{\lambda \mu}^{(1)}+S_{\lambda \mu}^{(2)}$

where

$S_{\lambda \mu}^{(0)}=\frac{1}{3} \delta_{\lambda \mu} S_{\nu \nu}^{f 0}$,

$S_{\lambda \mu}^{(1)}=\frac{1}{2}\left(S_{\lambda \mu}^{f 0}-S_{\mu \lambda}^{f 0}\right)$,

$S_{\lambda \mu}^{(2)}=\frac{1}{2}\left(S_{\lambda \mu}^{f 0}+S_{\mu \lambda}^{f 0}\right)-\frac{1}{3} \delta_{\lambda \mu} S_{\nu \nu}^{f 0}$,

where $S_{\lambda_{\mu}}^{(0)}$ is a weight $0^{+}$tensor which transforms as a scalar; $S_{\lambda \mu}^{(1)}$ is a weight $1^{+}$tensor which transforms like the rotations; and $S_{\lambda \mu}^{(2)}$ is a weight $2^{+}$tensor which transforms as a second-rank, symmetric, traceless tensor. This allows us to rewrite the two parts to the rate equation, $B$ and $C$, involving the electro-optical tensor

$$
\begin{aligned}
B= & -\frac{1}{5} \mathrm{i} j_{1}^{\prime}(-\mathrm{i} \gamma) \mathscr{E}\left[(3+p)\left(S_{\lambda z}^{(0)}+S_{\lambda z}^{(2)}\right) \mu_{\lambda}^{f 0}\right. \\
& \left.+(3 p-1) S_{\lambda \lambda}^{(0)} \mu_{z}^{f 0}\right]+5(p-1) S_{\lambda z}^{(1)} \mu_{\lambda}^{f 0} \\
& +\frac{1}{5} \mathrm{i} j_{3}^{\prime}(-\mathrm{i} \gamma) \mathscr{E}(3 p-1)\left[5\left(S_{z z}^{(0)}+S_{z z}^{(2)}\right) \mu_{z}^{f 0}\right. \\
& \left.-S_{\lambda \lambda}^{(0)} \mu_{z}^{f 0}-2\left(S_{\lambda z}^{(0)}+S_{\lambda z}^{(2)}\right) \mu_{\lambda}^{f 0}\right] \\
C= & \frac{1}{30} \mathscr{E}^{2}\left[10 p S_{\lambda \mu}^{(0)} S_{\lambda \mu}^{(0)}+5(1-p) S_{\lambda \mu}^{(1)} S_{\lambda \mu}^{(1)}\right. \\
& \left.+(3+p) S_{\lambda \mu}^{(2)} S_{\lambda \mu}^{(2)}\right] \\
& -\frac{1}{42} \mathscr{E}^{2} j_{2}^{\prime}(-\mathrm{i} \gamma)\left\{( p - 1 ) \left[15\left(S_{\lambda z}^{(0)}+S_{\lambda z}^{(2)}\right)\right.\right.
\end{aligned}
$$

$$
\begin{aligned}
& \times\left(S_{\lambda z}^{(0)}+S_{\lambda z}^{(2)}\right)+42\left(S_{\lambda z}^{(0)}+S_{\lambda z}^{(2)}\right) S_{\lambda z}^{(1)} \\
& \left.-5 S_{\lambda \mu}^{(1)} S_{\lambda \mu}^{(1)}\right]+(15-7 p)\left(3 S_{\lambda z}^{(1)} S_{\lambda z}^{(1)}\right. \\
& \left.-S_{\lambda \mu}^{(2)} S_{\lambda \mu}^{(2)}\right)+6(3 p-5) \\
& \left.\times\left(S_{z z}^{(0)}+S_{z z}^{(2)}\right) S_{\lambda \lambda}^{(0)}+5(5 p-9) S_{\lambda \mu}^{(0)} S_{\lambda \mu}^{(0)}\right\} \\
& +\frac{1}{70} \mathscr{E}^{2} j_{4}^{\prime}(-\mathrm{i} \gamma)(3 p-1)\left\{5 \left[\left(S_{z z}^{(0)}+S_{z z}^{(2)}\right)\right.\right. \\
& \times\left(S_{z z}^{(0)}+7 S_{z z}^{(2)}\right)-4\left(S_{\lambda z}^{(0)}+S_{\lambda z}^{(2)}\right) \\
& \left.\left.\times\left(S_{\lambda z}^{(0)}+S_{\lambda z}^{(2)}\right)+S_{\lambda \mu}^{(0)} S_{\lambda \mu}^{(0)}\right]+2 S_{\lambda \mu}^{(2)} S_{\lambda \mu}^{(2)}\right\},
\end{aligned}
$$

where the initial and final states have been dropped from the electro-optical parameter, and the subscript $z$ refers to a choice of the $z$ axis in the molecular frame for the direction of the permanent molecular dipole.

Together, eqs. (13), (23) and (24) constitute a complete set of rate equations, the properties of which we shall now investigate.

\section{Symmetry considerations}

At this stage we can establish a link between the six-fold classification scheme for field-induced absorption developed in our earlier paper [2], table 2. This scheme was based on the possible combinations of allowed weights $0^{+}, 1^{+}$and $2^{+}$of the electro-optical tensor for a particular transition; for clarity these are now referred to with a + sign to indicate that they result from $(\mathrm{E} 1 \times \mathrm{E} 1)$ coupling.

For the cases where the molecule possesses a permanent electric dipole moment the point group symmetry is restricted to the axial point groups $\mathrm{C}_{n}$

Table 1

The reduced spherical Bessel functions, $j_{n}^{\prime}(-i \gamma)$, defined in eq. (17) and their limits both at high $(\gamma \gg 1)$ and low $(\gamma \ll 1)$ field

\begin{tabular}{lll}
\hline Full expression for $j_{n}^{\prime}(-\mathrm{i} \gamma)$ & Low-field limit, $\gamma \ll 1$ & High-field limit, $\gamma \gg 1$ \\
\hline$j_{0}^{\prime}=1$ & $j_{0}^{\prime}=1$ & $j_{0}^{\prime}=1$ \\
$j_{1}^{\prime}=\mathrm{i}(1 / \gamma-\operatorname{coth} \gamma)$ & $j_{1}^{\prime}=-\mathrm{i}\left(\gamma / 3-\gamma^{3} / 45+2 \gamma^{5} / 945 \cdots\right)$ & $j_{1}^{\prime}=\mathrm{i}(1 / \gamma-1)$ \\
$j_{2}^{\prime}=-1-3 / \gamma^{2}+(3 / \gamma) \operatorname{coth} \gamma$ & $j_{2}^{\prime}=-\gamma^{2} / 15+2 \gamma^{4} / 315 \cdots$ & $j_{2}^{\prime}=-1+3 / \gamma-3 / \gamma^{2}$ \\
$j_{3}^{\prime}=\mathrm{i}\left[\left(1+15 / \gamma^{2}\right) \operatorname{coth} \gamma-6 / \gamma-15 / \gamma^{3}\right]$ & $j_{3}^{\prime}=\mathrm{i}\left(\gamma^{3} / 105-\gamma^{5} / 945 \cdots\right)$ & $j_{3}^{\prime}=\mathrm{i}\left(1-6 / \gamma+15 / \gamma^{2}-15 / \gamma^{3}\right)$ \\
$j_{4}^{\prime}=1+45 / \gamma^{2}+105 / \gamma^{4}-\left(10 / \gamma+105 / \gamma^{3}\right)$ & $j_{4}^{\prime}=\gamma^{4} / 945 \cdots$ & $j_{4}^{\prime}=1-10 / \gamma+45 / \gamma^{2}-105 / \gamma^{3}$ \\
& & \\
\hline
\end{tabular}


Table 2

The classification scheme developed for optically forbidden transitions in non-polar molecules

\begin{tabular}{ll}
\hline Class & Allowed weights \\
\hline (a) & $0^{+}, 1^{+}, 2^{+}$ \\
(b) & $1^{+}, 2^{+}$ \\
(c) & $0^{+}, 2^{+}$ \\
(d) & $2^{+}$ \\
(e) & $1^{+}$ \\
(f) & $0^{+}$ \\
\hline
\end{tabular}

and $C_{n v}$. The different weights can then be related to the following functions, the transformation properties of which are listed in most character tables

$S_{\lambda \mu}^{(0)} \Leftrightarrow x^{2}+y^{2}+z^{2}$,

$S_{\lambda \mu}^{(1)} \Leftrightarrow R_{x}, R_{y}, R_{z}$,

$\mu_{\lambda}^{f 0} \Leftrightarrow x, y, z$,

$S_{\lambda \mu}^{(2)} \Leftrightarrow 2 z^{2}-x^{2}-y^{2}, x^{2}-y^{2}, x y, x z, y z$.

Before proceeding to apply these properties, the number of possible cases can be reduced. If the final state for the transition belongs to the totally symmetric representation, then both $\mu_{z}^{f 0}$ and $S_{z z}^{f 0}$ will be non-zero. The latter has irreducible components $S_{z z}^{(0)}+S_{z z}^{(2)}$, so that any transition for which $S_{\lambda \mu}^{(0)}$ (weight $0^{+}$) is non-zero will also have finite values for $\mu_{\lambda}^{f 0}$ (weight $1^{-}$) and $S_{\lambda \mu}^{(2)}$ (weight $2^{+}$). Hence, case (f) of the previous paper (only weight $0^{+}$allowed) does not now occur.

There are then only three classes of optically allowed transitions, $\left(a^{\prime}\right),\left(b^{\prime}\right)$ and $\left(c^{\prime}\right)$, and three classes of optically forbidden transitions, (b), (d) and (e), the active components of which are shown in table 3. These two groups can immediately be experimentally distinguished since the latter appear as new features in the spectrum on application of the field.

\subsection{Optically allowed transitions}

$$
\begin{aligned}
\text { Case } & \left(a^{\prime}\right) \\
A= & \frac{1}{3}\left[1-j_{2}^{\prime}(-\mathrm{i} \gamma)(3 p-1)\right] \mu_{z}^{f 0} \mu_{z}^{f 0}, \\
B= & -\frac{1}{5} \mathrm{i} j_{1}^{\prime}(-\mathrm{i} \gamma) \mathscr{E}\left[(3+p)\left(S_{z z}^{(0)}+S_{z z}^{(2)}\right) \mu_{z}^{f 0}\right. \\
& \left.+(3 p-1) S_{\lambda \lambda}^{(0)} \mu_{z}^{f 0}\right] \\
& +\frac{1}{5} \mathrm{i} j_{3}^{\prime}(-\mathrm{i} \gamma) \mathscr{E}(3 p-1)\left[3\left(S_{z z}^{(0)}+S_{z z}^{(2)}\right) \mu_{z}^{f 0}\right. \\
& \left.-S_{\lambda \lambda}^{(0)} \mu_{z}^{f 0}\right] \\
C= & \frac{1}{30} \mathscr{E}^{2}\left[10 p S_{\lambda \mu}^{(0)} S_{\lambda \mu}^{(0)}+5(1-p) S_{\lambda \mu}^{(1)} S_{\lambda \mu}^{(1)}\right. \\
& \left.+(3+p) S_{\lambda \mu}^{(2)} S_{\lambda \mu}^{(2)}\right]-\frac{1}{42} \mathscr{E}^{2} j_{2}^{\prime}(-\mathrm{i} \gamma) \\
& \times\left\{( p - 1 ) \left[15\left(S_{z z}^{(0)}+S_{z z}^{(2)}\right)\left(S_{z z}^{(0)}+S_{z z}^{(2)}\right)\right.\right. \\
& \left.-5 S_{\lambda \mu}^{(1)} S_{\lambda \mu}^{(1)}\right]+(7 p-15) S_{\lambda \mu}^{(2)} S_{\lambda \mu}^{(2)}+6(3 p-5) \\
& \left.\times\left(S_{z z}^{(0)}+S_{z z}^{(2)}\right) S_{\lambda \lambda}^{(0)}+5(5 p-9) S_{\lambda \mu}^{(0)} S_{\lambda \mu}^{(0)}\right\} \\
& +\frac{1}{70} \mathscr{E}^{2} j_{4}^{\prime}(-\mathrm{i} \gamma)(3 p-1)\left\{5 \left[3\left(S_{z z}^{(0)}+S_{z z}^{(2)}\right)\right.\right. \\
& \left.\left.\times\left(S_{z z}^{(2)}-S_{z z}^{(0)}\right)+S_{\lambda \mu}^{(0)} S_{\lambda \mu}^{(0)}\right]+2 S_{\lambda \mu}^{(2)} S_{\lambda \mu}^{(2)}\right\}
\end{aligned}
$$

Case $\left(b^{\prime}\right)$

$$
\begin{aligned}
A= & \frac{1}{3}\left[1+j_{2}^{\prime}(-\mathrm{i} \gamma)(3 p-1)\right] \mu_{\psi}^{f 0} \mu_{\psi}^{f 0}, \\
B= & -\frac{1}{5} \mathrm{i} j_{1}^{\prime}(-\mathrm{i} \gamma) \mathscr{E}\left[(3+p) S_{\psi z}^{(2)} \mu_{\psi}^{f 0}\right. \\
& \left.+5(p-1) S_{\psi z}^{(1)} \mu_{\psi}^{f 0}\right] \\
& -\frac{1}{5} \mathrm{i} j_{3}^{\prime}(-\mathrm{i} \gamma) \mathscr{E}(3 p-1) 2 S_{\psi z}^{(2)} \mu_{\psi}^{f 0},
\end{aligned}
$$

Table 3

The characters of the six allowed classes of transition for polar molecules

\begin{tabular}{lll}
\hline Class & Allowed weights & Characters of the allowed transitions \\
\hline$\left(a^{\prime}\right)$ & $0^{+}, 1^{-}, 1^{+}, 2^{+}$ & $\mathrm{C}_{n} ; \mathrm{A}$ \\
$\left(\mathrm{b}^{\prime}\right)$ & $1^{-}, 1^{+}, 2^{+}$ & $\mathrm{C}_{2 \mathrm{v}} ; \mathrm{B}_{1}$ and $\mathrm{B}_{2}, \mathrm{C}_{2} ; \mathrm{B}, \mathrm{C}_{n}, \mathrm{C}_{n v}(n \geqslant 4) ; \mathrm{E}_{1}, \mathrm{C}_{3}$ and $\mathrm{C}_{3 \mathrm{v}} ; \mathrm{E}$ \\
$\left(\mathrm{c}^{\prime}\right)$ & $0^{+}, 1^{-}, 2^{+}$ & $\mathrm{C}_{n v} ; \mathrm{A}_{1}$ \\
(b) & $1^{+}, 2^{+}$ & $\mathrm{C}_{2 \mathrm{v}} ; \mathrm{A}_{2}$ \\
(d) & $2^{+}$ & $\mathrm{C}_{4 \mathrm{v}} ; \mathrm{B}_{1}$ and $\mathrm{B}_{2}, \mathrm{C}_{4} ; \mathrm{B}, \mathrm{C}_{n}, \mathrm{C}_{n v}(n>4) ; \mathrm{E}_{2}$ \\
(e) & $1^{+}$ & $\mathrm{C}_{n \mathrm{v}}(\mathrm{n} \geqslant 3) ; \mathrm{A}_{2}$ \\
\hline
\end{tabular}




$$
\begin{aligned}
C= & \frac{1}{30} \mathscr{E}^{2}\left[5(1-p) S_{\lambda \mu}^{(1)} S_{\lambda \mu}^{(1)}+(3+p) S_{\lambda \mu}^{(2)} S_{\lambda \mu}^{(2)}\right] \\
& -\frac{1}{42} \mathscr{E}^{2} j_{2}^{\prime}(-\mathrm{i} \gamma)\left[( 1 - p ) \left(15 S_{\psi z}^{(2)} S_{\psi z}^{(2)}\right.\right. \\
& \left.+42 S_{\psi z}^{(2)} S_{\psi z}^{(1)}-5 S_{\lambda \mu}^{(1)} S_{\lambda \mu}^{(1)}\right) \\
& \left.+(15-7 p)\left(3 S_{\psi z}^{(1)} S_{\psi z}^{(1)}-S_{\lambda \mu}^{(2)} S_{\lambda \mu}^{(2)}\right)\right] \\
& +\frac{1}{35} \mathscr{E}^{2} j_{4}^{\prime}(-\mathrm{i} \gamma)(3 p-1)\left(S_{\lambda \mu}^{(2)} S_{\lambda \mu}^{(2)}-10 S_{\psi z}^{(2)} S_{\psi z}^{(2)}\right)
\end{aligned}
$$

where $\psi$ is the set $\{x, y\}$ or $\{(x+\mathrm{i} y),(x-\mathrm{i} y)\}$ as appropriate.

\section{Case $\left(c^{\prime}\right)$}

Eqs. (29), (30) and

$$
\begin{aligned}
C= & \frac{1}{30} \mathscr{E}^{2}\left[10 p S_{\lambda \mu}^{(0)} S_{\lambda \mu}^{(0)}+(3+p) S_{\lambda \mu}^{(2)} S_{\lambda \mu}^{(2)}\right] \\
& -\frac{1}{42} \mathscr{E}^{2} j_{2}^{\prime}(-\mathrm{i} \gamma)\left[15(p-1)\left(S_{z z}^{(0)}+S_{z z}^{(2)}\right)\right. \\
& \times\left(S_{z z}^{(0)}+S_{z z}^{(2)}\right)+(7 p-15) S_{\lambda \mu}^{(2)} S_{\lambda \mu}^{(2)} \\
& +6(3 p-5)\left(S_{z z}^{(0)}+S_{z z}^{(2)}\right) S_{\lambda \lambda}^{(0)} \\
& \left.+5(5 p-9) S_{\lambda \mu}^{(0)} S_{\lambda \mu}^{(0)}\right] \\
& +\frac{1}{70} \mathscr{E}_{4}^{\prime}(-\mathrm{i} \gamma)(3 p-1)\left\{5 \left[3\left(S_{z z}^{(0)}+S_{z z}^{(2)}\right)\right.\right. \\
& \left.\left.\times\left(S_{z z}^{(2)}-S_{z z}^{(0)}\right)+S_{\lambda \mu}^{(0)} S_{\lambda \mu}^{(0)}\right]+2 S_{\lambda \mu}^{(2)} S_{\lambda \mu}^{(2)}\right\} .
\end{aligned}
$$

From table 3 it is obvious that both the $\left(a^{\prime}\right)$ and $\left(c^{\prime}\right)$ types of transition cannot occur in the same molecule; thus it is important only to be able to separate $\left(b^{\prime}\right)$ from $\left(a^{\prime}\right)$ or $\left(c^{\prime}\right)$ classes of transition. It is not possible, however, to prescribe a general experimental procedure to distinguish between these classes. Generally the rate will be dominated by the pure optical pathway, whilst the $j_{2}^{\prime}(-\mathrm{i} \gamma)$ term of $A$, the $j_{1}^{\prime}(-\mathrm{i} \gamma)$ term of $B$ and the zeroth term of $C$ may all be equally important and hence only separable on the basis of their different temperature dependence.

\subsection{Optically forbidden transitions}

In the physically more interesting cases, where the transition is optically forbidden, the results are appreciably simplified since $A=B=0$, leaving the following expressions for $C$;
Case $(b)$

$$
\begin{aligned}
C= & \frac{1}{30} \mathscr{E}^{2}\left[5(1-p) S_{\lambda \mu}^{(1)} S_{\lambda \mu}^{(1)}+(3+p) S_{\lambda \mu}^{(2)} S_{\lambda \mu}^{(2)}\right] \\
& +\frac{1}{42} \mathscr{E}^{2} j_{2}^{\prime}(-\mathrm{i} \gamma)\left[5(p-1) S_{\lambda \mu}^{(1)} S_{\lambda \mu}^{(1)}\right. \\
& \left.+(15-7 p) S_{\lambda \mu}^{(2)} S_{\lambda \mu}^{(2)}\right]+\frac{1}{35} \mathscr{E}^{2} j_{4}^{\prime}(-\mathrm{i} \gamma) \\
& \times(3 p-1) S_{\lambda \mu}^{(2)} S_{\lambda \mu}^{(2)} .
\end{aligned}
$$

Case $(d)$

$$
\begin{aligned}
C= & \frac{1}{30} \mathscr{E}^{2}(3+p) S_{\lambda \mu}^{(2)} S_{\lambda \mu}^{(2)}-\frac{1}{42} \mathscr{E}^{2} j_{2}^{\prime}(-\mathrm{i} \gamma) \\
& \times(7 p-15) S_{\lambda \mu}^{(2)} S_{\lambda \mu}^{(2)} \\
& +\frac{1}{35} \mathscr{E}^{2} j_{4}^{\prime}(-\mathrm{i} \gamma)(3 p-1) S_{\lambda \mu}^{(2)} S_{\lambda \mu}^{(2)} .
\end{aligned}
$$

Case (e)

$$
\begin{aligned}
C= & \frac{1}{6} \mathscr{E}^{2}(1-p) S_{\lambda \mu}^{(1)} S_{\lambda \mu}^{(1)}-\frac{1}{42} \mathscr{E}^{2} j_{2}^{\prime}(-\mathrm{i} \gamma) \\
& \times\left[5(1-p) S_{\lambda \mu}^{(1)} S_{\lambda \mu}^{(1)}\right] .
\end{aligned}
$$

Case (b) only arises for transitions of $\mathrm{A}_{2}$ symmetry in $C_{2 v}$ molecules, where there is no possibility of transitions of types (d) or (e). It is also immediately obvious that by arranging for the polarisation and static field vectors to be parallel, then for case (e) transitions the induced features should completely disappear. Hence it is a comparatively simple matter to distinguish the class to which any optically forbidden transition belongs.

\subsection{No permanent dipole moment}

Finally we note that in cases where the molecule possesses no permanent moment, all terms involving $j_{n}^{\prime}(-\mathrm{i} \gamma)$ vanish for $n>0$, leaving the isotropic result. The appropriate rate equation is thus

$$
\begin{aligned}
\Gamma= & \left(\pi I \rho_{\mathrm{f}} / \epsilon_{0} \hbar c\right)\left\{\frac{1}{3}\left(\mu_{\lambda}^{f 0} \mu_{\lambda}^{f 0}\right)+\frac{1}{30} \mathscr{E}^{2}\left[10 p S_{\lambda \mu}^{(0)} S_{\lambda \mu}^{(0)}\right.\right. \\
& \left.\left.+5(1-p) S_{\lambda \mu}^{(1)} S_{\lambda \mu}^{(1)}+(3+p) S_{\lambda \mu}^{(2)} S_{\lambda \mu}^{(2)}\right]\right\}
\end{aligned}
$$

The leading field-independent term is also polarisation-independent, and the correction term has a polarisation dependence which has previously been investigated [2]. 


\section{Conclusion}

In this paper we have provided the detailed equations to describe the effect of a static electric field on the absorption spectra of a molecular fluid, caused both by orientational anisotropy and the first-order electro-optical pathway. In the case of optically (E1)-allowed transitions, detailed study of the temperature dependence of the absorption rate may enable the various rate contributors $A, B$ and $C$ to be specifically identified. Also for the case of (E1)-forbidden transitions we have shown that by selective polarisation experiments it is possible to distinguish between the allowed types of transitions.

It is pertinent to consider the likely magnitudes of the contributors to the rate equations that we have derived. We have previously demonstrated that the intensity of electro-optically allowed transitions is in the same region as that typically associated with two-photon spectroscopy [2]. To estimate more precisely the orders of magnitude of our results, we can consider an experimentally feasible [8] electric field strength of $10^{8} \mathrm{~V} \mathrm{~m}^{-1}$, typical permanent and transition dipole moments of $1 \mathrm{D}$ and a value for the components of the tensor $S$ estimated from the molecular polarisability, which has a similar structure and is typically of the order $10^{-39} \mathrm{~J}^{-1} \mathrm{C}^{2} \mathrm{~m}^{2}$. In this case the magnitude of $\gamma$ at STP is 0.08 , and the leading contribution to $B$ will be of the same magnitude as that of $C$ and the $j_{2}^{\prime}(-\mathrm{i} \gamma)$ term of $A$, whilst the leading field-independent (E1) term in $A$ will be between three and four orders of magnitude larger. Despite the small size of the field-induced effects on (E1)-allowed transitions, their measurement is well within the bounds of experimental feasibility by square-wave modulation of the electric field coupled with phase-sensitive detection.

\section{Acknowledgement}

One of us, BSS, gratefully acknowledges financial support from the Science and Engineering Research Council.

\section{References}

[1] G.E. Stedman, Advan. Phys. 34 (1985) 518.

[2] D.L. Andrews and B.S. Sherborne, Chem. Phys. 88 (1984) 1.

[3] D.L. Andrews and M.J. Harlow, Phys. Rev. A29 (1984) 2796.

[4] D.L. Andrews and B.S. Sherborne, J. Phys. B, to be published.

[5] D.P. Craig and T. Thirunamachandran, Molecular quantum electrodynamics (Academic Press, New York, 1984).

[6] K. Yamaoka and E. Charney, J. Am. Chem. Soc. 94 (1972) 8963.

[7] I.M. Gel'fand, R.A. Minlos and Z.Ya. Shapiro, in: Representations of the rotation and Lorentz groups and their applications (Pergamon Press, Oxford, 1963) p. 64.

[8] P. van Pelt and E.E. Havinga, in: Nonlinear behaviour of molecules, atoms and ions in electric, magnetic or electromagnetic fields, ed. L. Neel (Elsevier, Amsterdam, 1979) p. 291. 